\title{
New Analytical Methods for the Surface/ Interface and the Micro-Structures in Advanced Nanocomposite Materials by Synchrotron Radiation
}

\author{
K. Nakamae ${ }^{\star}$, J. Matsui, K. Yokoyama, Y. Urushihara, S. Kuwamoto, \\ L. Li and S. Takeda \\ Hyogo-Prefecture Synchrotron Radiation Nanotechnology Laboratory \\ 1-490-2, Kouto, Shingu-cho, Tatsuno-shi, Hyogo, 679-5165 Japan
}

\section{ARTICLE INFO}

Article history:

Received 11 October 2010

Received in Revised form 13 December 2010

Accepted 23 December 2010

Keywords:

Interfaces

Microstructures

Nanocomposite

Nanostructures

Surfaces

Synchrotron radiation

\begin{abstract}
A B S T R A C T
Analytical methods of surface/interface structure and micro-structure in advanced nanocomposite materials by using the synchrotron radiation are introduced. Recent results obtained by the energy-tunable and highly collimated brilliant X-rays, in-situ wide angle/small angle X-ray diffraction with high accuracy are reviewed. It is shown that small angle X-ray scattering is one of the best methods to characterize nanoparticle dispersibility, filler aggregate/agglomerate structures and in-situ observation of hierarchical structure deformation in filled rubber under cyclic stretch. Grazing Incidence(small and wide angle) X-ray Scattering are powerful to analyze the sintering process of metal nanoparticle by in-situ observation as well as the orientation of polymer molecules and crystalline orientation at very thin surface layer $(\mathrm{ca} 7 \mathrm{~nm})$ of polymer film. While the interaction and conformation of adsorbed molecule at interface can be investigated by using high energy X-ray XPS with Enough deep position (ca 9 micron $\mathrm{m}$ ).
\end{abstract}

(C) 2010 Atom Indonesia. All rights reserved

\section{INTRODUCTION}

It is very important to make clear the surface/interface and micro-structures in composite materials not only for the industrial development of the nanocomposite materials but also material science. The analytical methods of surface/interface structure and micro-structure by the synchrotron radiation (SR) have several advantages as compared with conventional analytical equipments since SPring- 8 can provide light of about one billion times more brilliant than conventional X-ray sources. SR is extremely powerful light for studying the structure of materials at the atomic, electronic levels and physical and chemical processes.

Development of nanoparticle composites is strongly supported by highly precise surface/interface and micro-structures analysis and also by in-situ observation of composite behavior. These procedure can be performed by newly developed methods combined with synchrotron radiation X-rays.

\footnotetext{
* Corresponding author.

E-mail address: nakamae@hyogosta.jp ( K. Nakamae )
}

In this paper, we introduce some of recent results obtained by using the energy-tunable and highly collimated brilliant X-rays, in-situ wide angle/small angle $\mathrm{X}$-ray diffraction with high accuracy.

\section{EXPERIMENTAL METHODS}

SPring-8 is the largest third-generation synchrotron radiation facility in the world. Fig. 1 shows SPring-8 and the synchrotron radiation spectrum [1].

Table 1. Analytical methods for the surface/Interface and micro-structure in advanced nanocomposite structures by synchrotron radiation $\mathrm{X}$-ray

Small-angle X-ray scattering (SAXS)

Ultra-Small-angle X-ray scattering (USAXS)

Grazing incidence small angle X-ray scattering (GI-SAXS)

Wide-angle X-ray scattering (WAXS)

Grazing incidence X-ray diffraction (GI-XRD)

$\mathrm{X}$-ray absorption fine structure(XAFS)

$\mathrm{X}$-ray reflectivity $(\mathrm{XR})$

High-energy X-ray photoelectron spectroscopy (HEPES) 
The light of SPring- 8 is composed by the wide range spectrum from the Infrared to the hard X-ray. The methods, which can be used in the SR science are summarized in Table 1.
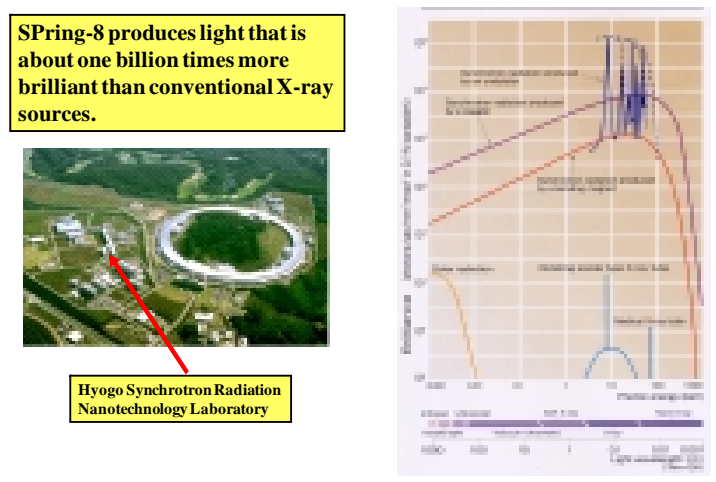

Fig. 1. Synchrotron radiation spectra of SPring-8 Brilliance and Wavelength.

\section{Small Angle X-ray Scattering (SAXS)}

The in-situ small angle X-ray scattering (SAXS) measurement is one of the best methods for the analysis of microstructure of nanocomposite and nucleation process of nanoparticle. The Hyogo Prefecture Nanotechnology Laboratory designed new small angle X-ray scattering system in BL08B2 for this purpose.

\section{RESULTS AND DISCUSSION}

\section{SAXS for nanoparticle formation process}

By using this method we can analyze the nanoparticle formation process. Fig. 2 shows one of experimental results. Silver metal nanoparticle can be obtained from the silver ions solution by chemical reaction.

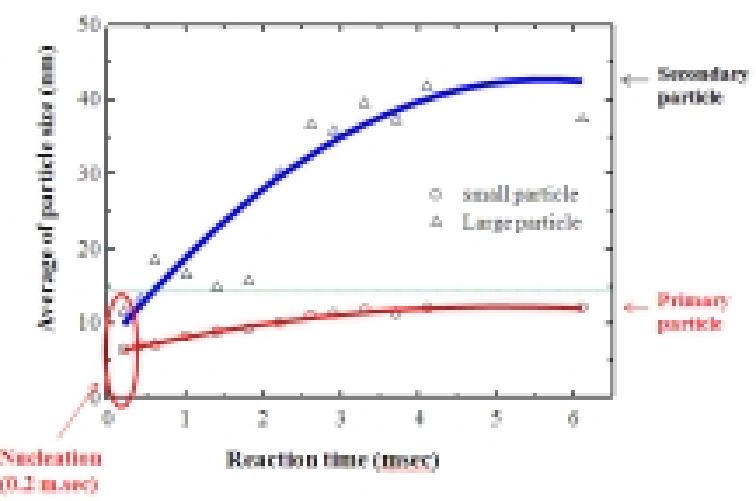

Fig. 2. SAXS in-situ observation of metal nanoparticle Nucleation (particle size and reaction time).
From Fig. 2 we can conclude that metal ions nucleate particles in a $0.2 \mathrm{msc}$ then grow up to primary nanoparticle in $3 \mathrm{msec}$ at first stage then a primary particle (ca $10 \mathrm{~nm}$ ) goes to aggregate to secondary particles in a $6 \mathrm{msc}$. with increasing a diameter (ca $30 \sim 40 \mathrm{~nm}[2]$.

\section{SAXS for rubber filled with spherical silica}

Recently the high performance tire has received much attention. For this purpose it is important to study the mechanism of reinforcement by filler in the rubber. In order to investigate the structural change of filler network the real time wide range structural scale observation has been required.

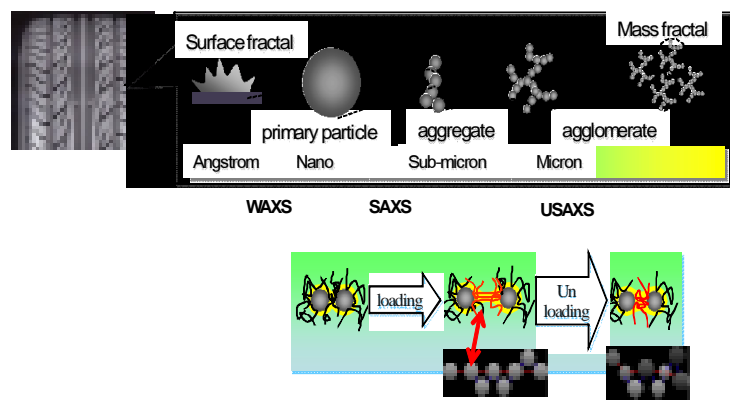

Fig. 3. The hierarchical structure of fillers in rubber.

Kishimoto et al proposed the hierarchical structures in rubber shown in Fig. 3 and has reported many research by using synchrotron radiation X-ray [3].

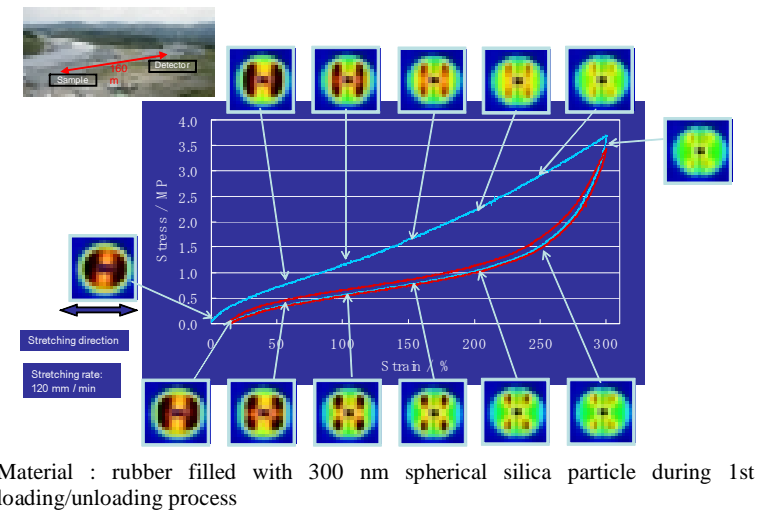

Fig. 4. USAXS image under cyclic stretch obtained in-situ observation by synchrotron radiation.

The structural change is caused by the interaction between filler and rubber under the loading and unloading. Therefore in-situ observation is the important method to the analysis for the reinforcement mechanism (Fig. 4).

From these results, the hierarchy of filled rubber, deformation of hierarchical structure and also mechanism of reinforcement can be discussed. 


\section{Grazing Incidence X-ray Small Angle X-ray Scattering (GI-SAXS)}

SEM is the very powerful tool to analyze the surface structure but it is difficult to observe the phase transition process. However, GI-SAXS can analyze the surface structure in the nanometer depth and the sintering process of nano-metal particles. Figure 5 shows the results of sintering process of gold nano-metal particles [4]. The in-situ observation of nanoparticle sintering process by using GI-SAXS can make clear the mechanism of particle break down to thin metal layer under the melting point of metal.
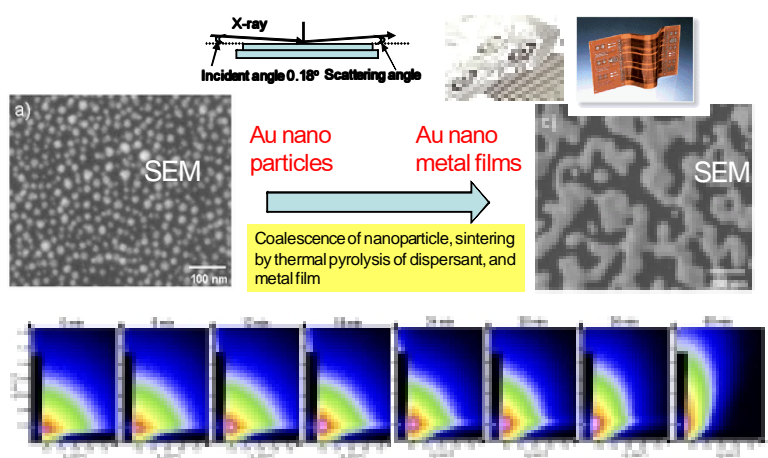

Typical 2D GI-SAXS patterns of Au nanoparticle paste to nano metal film at $280^{\circ} \mathrm{C}$

Fig. 5. Application of GI-SAXS to understand sintering process of nano metal particles.

\section{Grazing Incidence X-ray Scattering (GI-XRD}

If the incident angle of X-ray become smaller than the critical angle of X-ray can not penetrate into the materials. X-ray scattering is obtained from ca $7 \mathrm{~nm}$ depth surface layer.

The very thin surface layer (less than $7 \mathrm{~nm}$ ) structure analysis is very important to develop for the liquid crystal display devices. Rubbed surface structure of polyimide film is the most important key for the devices. However rubbed surface structure of polyimide has been unsolved problems in a long time.

Figure 6 shows the results obtained by parallel scanning to the rubbing direction and normal to the rubbing direction by using GI-XRD [5]. From this result, (002) diffraction can be observed only in parallel scanning and not in normal scanning. Therefore, it is clear that the polyimide molecules in the nm surface layer line parallel to the rubbing direction. This degree of molecular orientation of polyimide is the important key factor for the quality of FPD [5].
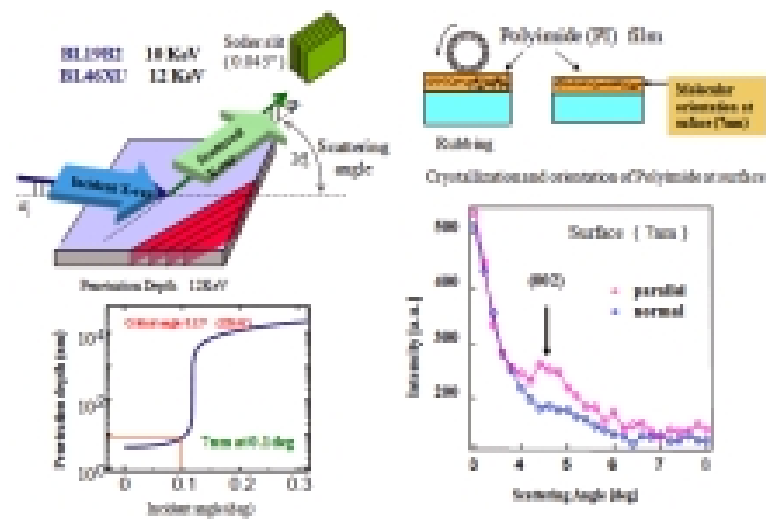

Fig. 6. Grazing incidence X-ray scattering (GI-XRD) for liquid crystal display device.

\section{High-energy X-ray Photoelectron Spectroscopy (High-energy XPS or HEPES)}

The conventional Photoelectron Spectroscopy (XPS) gives us very important information at the surface. However, the probing depth of conventional XPS is limited about $4 \mathrm{~nm}$ depth at the surface. On the other hand, X-ray photoelectron spectroscopy by synchrotron radiation gives us useful information about the interfacial structures under the surface because the probing depth of HEPES is about several micron $m$ [6]. High-energy $X$-ray by synchrotron radiation $(10 \mathrm{keV})$ can generate enough photoelectrons even at deep position ( ca 9 micron $\mathrm{m}$ ).

Figure 7 shows experimental results of High energy XPS data for phosphor 1s states of the thin lubricant PFPE $(\mathrm{A} 20 \mathrm{H})$ on the hard disk [6]. Therefore it is possible to make clear the electron state of elements in adsorbed molecule at interface. From this data, the conformation of adsorbed lubricants and the interaction at the hard disk surface are estimated as shown in Fig. 7 [7].

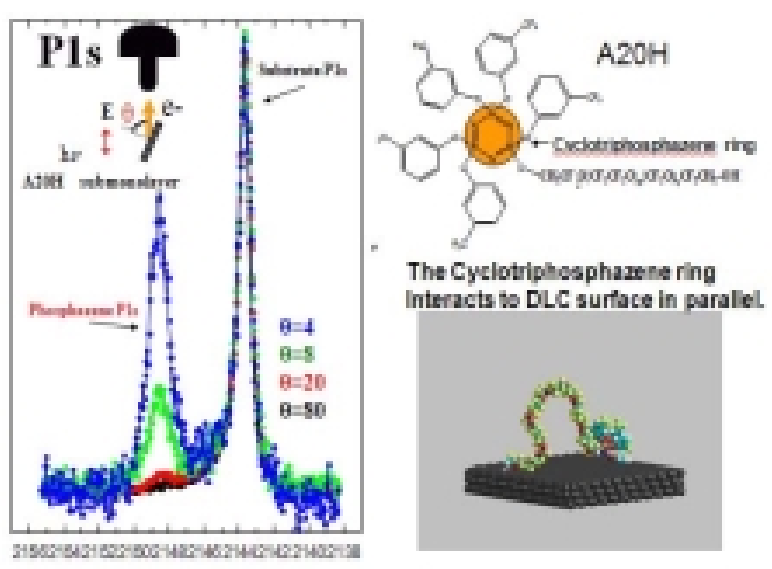

Fig. 7. High-energy XPS profile of phosphor-1s photoelectrons from $\mathrm{A} 2 \mathrm{OH}$ adsorbed on the DLC surface. 


\section{CONCLUSIONS}

Both SAXS and USAXS are best methods for the characterization of nanoparticle dispersibility, filler aggregate/ agglomerate structures and in-situ observation of hierarchical structure deformation in filled rubber under cyclic stretch. GI-SAXS can be used to analyze the sintering process of metal nanoparticle by in-situ observation. GI-XRD can be used analyze orientation of polymer molecules and crystalline orientation at very thin surface layer (ca $7 \mathrm{~nm}$ ) of polymer film. High energy X-ray XPS can be applied to investigate the interaction and conformation of adsorbed molecule at interface.

\section{REFERENCES}

1. SPring-8 Public relations office.

2. K. Hata, Japan Science and Technology Agency
Project, Collaboration of regional entities for the advancement of Technical excellence, 11, Tokyo (2008).

3. H. Kishimoto, Y. Shinohara, Y. Amamiya, K. Inoue, Y. Suzuki, A. Takeuchi, K. Uesugi and N. Yagi: Rubber Chemistry \& Technology, IRC 2005,Oct. Yokohama 81 (4) (2008) 541.

4. Y. Urushihara, L. Li, S. Kuwamoto, Y. Yokoyama, J. Matsui and K. Nakamae: Annual Meeting of the Japan Society of Colour Material (2009).

5. I. Hirosawa, N. Sasaki and H. Kimura, J. Appl. Phys. 38 (1999) 583.

6. E. Ikenaga et al., J. Electron Spectrosc. Rel. Phenom. 491 (2005)144.

7. Y. Sakane, A. Wakabayashi and T. Hirano, ITC Kobe, May 31 (2005). 